This item was submitted to Loughborough's Research Repository by the author.

Items in Figshare are protected by copyright, with all rights reserved, unless otherwise indicated.

\title{
Dipole-slot-dipole metasurfaces
}

\section{PLEASE CITE THE PUBLISHED VERSION}

http://dx.doi.org/10.1049/iet-map.2015.0849

\section{PUBLISHER}

(c) The Institution of Engineering and Technology (IET)

\section{VERSION}

AM (Accepted Manuscript)

\section{PUBLISHER STATEMENT}

This work is made available according to the conditions of the Creative Commons Attribution-NonCommercialNoDerivatives 4.0 International (CC BY-NC-ND 4.0) licence. Full details of this licence are available at: https://creativecommons.org/licenses/by-nc-nd/4.0/

\section{LICENCE}

CC BY-NC-ND 4.0

\section{REPOSITORY RECORD}

Bukhari, Syed S., W.G. Whittow, J.C. Vardaxoglou, and Stefano Maci. 2019. "Dipole-slot-dipole Metasurfaces". figshare. https://hdl.handle.net/2134/23000. 


\title{
Dipole-slot-dipole metasurfaces
}

ISSN 1751-8725

Received on 31st December 2015

Revised on 1st July 2016

Accepted on 30th August 2016

doi: 10.1049/iet-map.2015.0849

www.ietdl.org

\author{
Syed Sheheryar Bukhari ${ }^{凶}$, William G. Whittow ${ }^{1}$, John C. Vardaxoglou ${ }^{1}$, Stefano Maci ${ }^{2}$ \\ ${ }^{1}$ Wolfson School of Mechanical, Electrical and Manufacturing Engineering, Loughborough University, Loughborough, UK \\ ${ }^{2}$ Department of Information Engineering, University of Siena, Siena, Italy \\ $凶$ E-mail: s.s.bukhari@lboro.ac.uk
}

\begin{abstract}
A complementary frequency selective surface (CFSS) can be formed on the basis of Babinet's principle. It consists of an array of slots separated from an array of dipoles by a thin dielectric substrate. This study shows that by adding an extra layer of dipoles to a CFSS capacitance can be added to the structure, which leads to a decrease in its resonant frequency. This new structure is called a dipole-slot-dipole metasurface (MTS) and it has unit-cell dimensions of $\lambda / 10 \times \lambda / 10 \times \lambda / 333$, where $\lambda$ is representing the free space wavelength. The dipole-slot-dipole MTS has been fabricated and measured. The study also reports on its equivalent circuit; and the effects of the length of the dipoles on the added layer and their alignment on the pass band resonant frequency of the dipole-slot-dipole MTS.
\end{abstract}

\section{Introduction}

Metasurfaces (MTSs) are two dimensional counterparts of metamaterials that occupy comparatively smaller space than the three-dimensional metamaterials thus offering a possibility of less lossy structures. MTSs, also characterised as metafilms in the literature may possess individual elements resonating at a wavelength larger than the physical size of these elements. When the resonant frequency wavelength is comparable to the periodicity of the structure, higher order propagating Floquet modes need to be considered; therefore MTSs are designed such that their individual elements or 'individual scatterers' are much smaller in size, but still resonant, thus allowing them to have novel electromagnetic properties $[1,2]$. In this paper, the size of a coupled complementary MTS is reduced by adding an additional layer of dipoles. This dipole layer adds additional capacitance to the structure, thus decreasing the unit cell size at resonance. This miniaturisation makes the response of three-layer MTS very local and also allows packing a large number of unit cells over a small space.

MTSs offer a variety of potential applications for example, open waveguides for terahertz range, miniaturised cavity resonators, switches, etc. [1, 3]. MTSs have been proposed for medical body area networks as well, by improving the performance of a low-profile monopole antenna. This truncated MTS, in contrast to artificial magnetic conductors, also contributed to the radiation from the antenna, thus increasing the gain and the front to back ratio [4]. Two-layered MTSs loaded with resistors have been demonstrated to reduce the radar cross-section (RCS) of a stacked antenna array, thus offering an alternative to radar absorbing material (RAM). The antenna was sandwiched between these two MTSs; the metafilm above the antenna reduced the RCS for out of band frequencies, while the one below reduced the RCS for the in band frequencies [5].

MTSs have also been successfully used to design and manufacture high-gain holographic antennas [6, 7]. In [8], multi-layered MTSs with sub-wavelength profile were used as partial reflective surfaces to design a high-gain antenna with an enhanced bandwidth.

Non-linear MTSs have been deployed to protect the sensitive electronics and reduce the interference on a shared platform. Non-linearity was introduced by diodes and capacitors, which were incorporated in the design process for response control. This surface absorbed the high-powered radio frequency signals, while causing a minimal distortion to the low-powered ones [9]. MTSs have also found potential applications in optics and their property of phase discontinuity has been exploited to tailor wavefronts for optical waves [10]. In [11], Huygens MTSs, which do not reflect any energy for an arbitrary angle of incidence, have been presented. A circuit model for a Huygens MTS using a lattice network has been reported in [12].

MTSs can also improve the performance of horn antennas. In [13], a MTS designed using a genetic algorithm has been used as an inner surface for a conical horn and the cross-polar levels and the side lobe levels have been improved over the entire $\mathrm{Ku}$ band. A similar approach was applied to improve the performance of a hybrid mode square horn antenna [14].

\section{Complementary MTSs}

A complementary frequency selective surface (CFSS) is constructed by a thin sheet of dielectric with a periodic array of slots on its one side and an array of dipoles (its Babinet's complement) on its other side. In order to enhance the coupling of both layers for one polarisation, they are rotated by $90^{\circ}$ with respect to each another. When the dielectric is extremely thin, a strong coupling occurs between the dipoles and the slots, which considerably decreases the resonant frequency of the CFSS [15]. This causes a reduction in the size of the individual unit cell in terms of wavelength, thus we classify this structure as a MTS. The small individual scatterer size also introduces locality and makes the response of the structure insensitive to the changes in the incidence angle of the illuminating plane wave. Other type of complementary MTSs has been investigated in literature; surface waves can be supported on self-complementary MTSs [16]. MTSs with complementary split ring resonators in different orientations exhibited either a band stop or band pass behaviour [17]. Checkerboard MTSs with adjacently connected patches, have been shown to support a quasi-Transverse Electro-magnetic (TEM) mode [18].

\section{Dipole-slot-dipole MTSs}

The work presented in this paper focuses on decreasing the resonant frequency of a dipole-slot MTS by adding an additional layer of dielectric backed dipoles. A periodic array of complementary MTS shows band-pass behaviour, thus indicating that the slot layer is the dominant component of this structure at resonance. The dipole, 


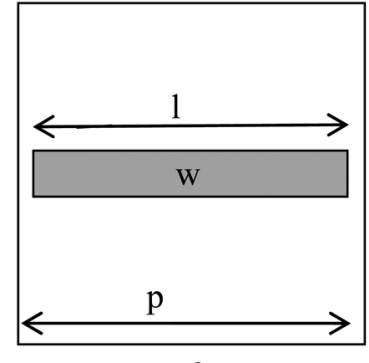

a

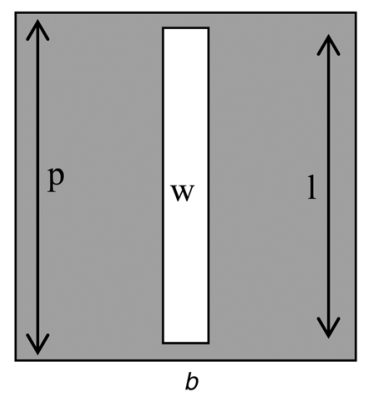

Fig. 1 Individual basic elements for dipole-slot MTS and dipole-slot-dipole MTS (length $(l)=4.5 \mathrm{~mm}$, width $(w)=0.5 \mathrm{~mm}$, periodicity $(p)=5 \mathrm{~mm}$ ) green shows the presence of metal (redrawn as [19])

$a$ Slot unit cell (layer 2)

$b$ Dipole unit cell (layers 1 and 3 )

which is naturally a capacitive element, adds extra capacitance to this slot layer, which significantly lowers its resonant frequency. The new structure has the configuration of dipole-slot-dipole and we call it a dipole-slot-dipole MTS. Fig. 1 shows the individual building blocks of dipole-slot MTS and dipole-slot-dipole MTS. A dipole-slot-dipole MTS is shown in Fig. 2. Each layer of the slot and the dipole is separated is by a thin sheet of a dielectric material.

The comparison between a dipole-slot-dipole MTS with a dipole-slot MTS is shown in Fig. 3. Both structures are simulated in CST Microwave Studio using periodic boundary conditions under normal incidence with $E$-field polarised along the dipoles. The periodic cell is $5 \times 5 \mathrm{~mm}$; the dipoles and slots are $0.5 \mathrm{~mm}$ wide and $4.5 \mathrm{~mm}$ long. The dielectric used in the simulations is $44 \mu \mathrm{m}$ thick, with a dielectric constant of 3 and a loss tangent of 0.01 . The dipole-slot MTS showed a resonance at $7.41 \mathrm{GHz}$. The dipole-slot-dipole MTS, which has an extra layer of identical dielectric backed dipoles added to the slot side, as shown in Fig. 2, showed a resonance at $5.96 \mathrm{GHz}$ (wavelength $\lambda=5 \mathrm{~cm}$ ); the periodicity at resonance is $\lambda / 10$. This decrease in the resonant frequency is due to the additional capacitance added to the dipole-slot MTS by the newly added dipole layer (third layer). The additional capacitance also causes an increase in the $q$-factor, thus decreasing the bandwidth of the structure. It should be noted that, for the results shown in Fig. 3, the dielectric on which the dipoles are placed is exactly the same as the one used for dipole-slot MTS.

The behaviour of the dipole-slot-dipole MTS can be illustrated by an equivalent circuit model. Figs. $4 a$ and $b$ show the equivalent

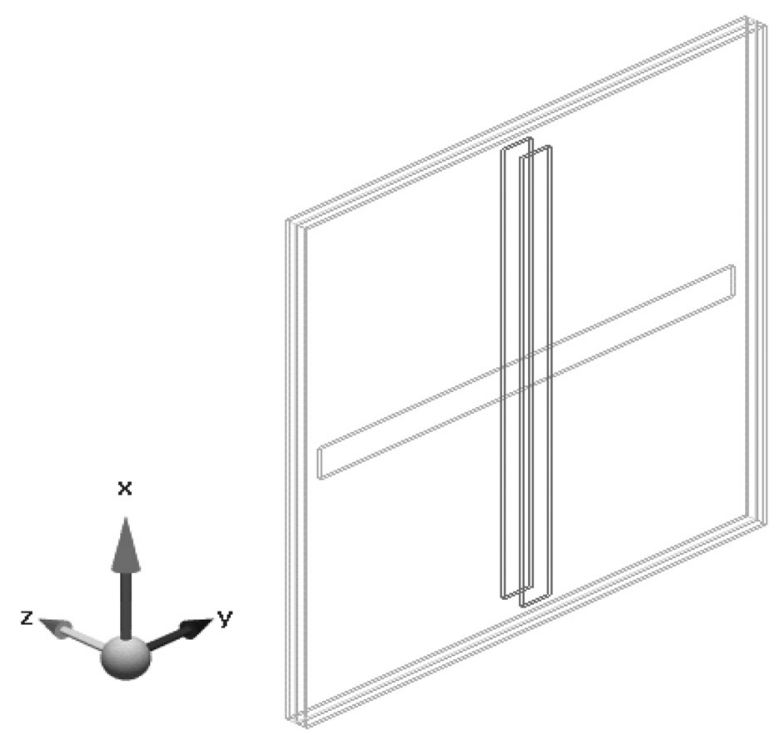

Fig. 2 Dipole-slot-dipole MTS unit cell showing grey slot placed among two red dipoles, each layer of dipoles and slot is separated by a thin dielectric (redrawn as in [19])

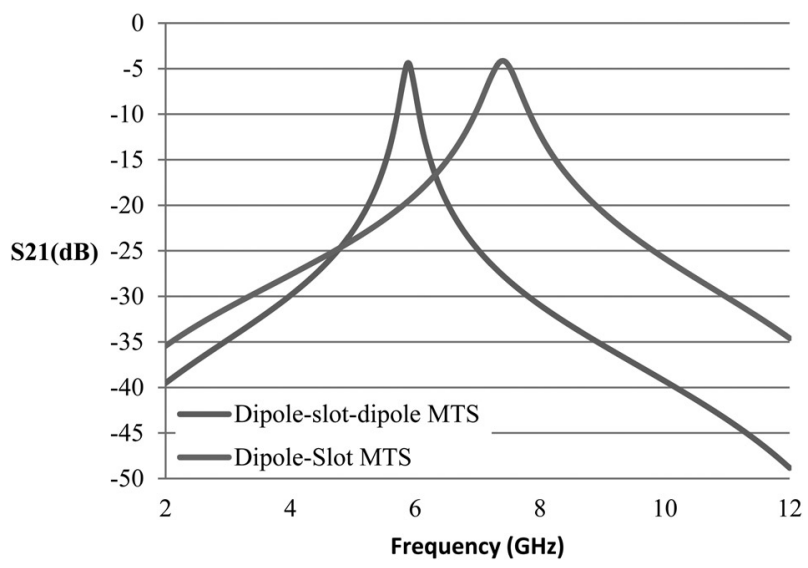

Fig. 3 Simulated comparison between the dipole-slot-dipole MTS and the dipole-slot MTS

circuits for both the dipole-slot-dipole MTS and the dipole-slot MTS. The first series $L C$ branch of the equivalent circuit represents the first dipole layer, with $C_{\text {dipole }}$ and $L_{\text {dipole }}$ being the capacitive and inductive part of the dipoles. The slot layer, as shown in Figs. $4 a$ and $b$, is represented by the parallel $L C$ circuit. $L_{\text {slot }}$ and $C_{\text {slot }}$ are the inductive and the capacitive parts of the slot layer. Since a periodic layer of dipoles is band stop so it is represented by a series $L C$ circuit; while a slot layer is band pass, thus represented by a parallel $L C$ circuit.

The coupling between the two layers is represented by a shunt capacitance $C_{1}$. It is the presence of this capacitance, which causes a decrease in the resonant frequency of the individual dipole-slot structure, in comparison with the resonant frequency of a single layer of either the dipoles or the slots. Because the resulting response of the dipole-slot-dipole MTS (and dipole-slot MTS) is that of a band pass (spatial filter); therefore the low-frequency dominant part of the dipoles circuit (capacitance) can be used to represent the couplings between the layers. The values of $C_{\text {dipole }}$ can be calculated from the relations given in $[16,20,21]$.

$$
C_{\text {dipole }}=\frac{2 \varepsilon_{0} p}{\pi} \ln \left(\frac{1}{\sin (\pi p / 2 d)}\right)
$$

where $C_{\text {dipole }}$ is the capacitance for the layer of dipoles in isolation in free space, $\varepsilon_{0}$ is the permittivity of free space, $p$ is the periodicity of the structure and $d$ is the distance between the two dipoles. The presence of the dielectric in between the elements is negligible due to its extremely small thickness and can be accounted for in the additional equivalent circuit element $C_{1}$.

The value for the inductance of the slot can then be ascertained by using the Babinet's principles, as shown in [16]

$$
\sqrt{\frac{L_{\text {slot }}}{C_{\text {dipole }}}}=\frac{Z_{0}}{2}
$$

with $L_{\text {slot }}$ being the inductance of the layer of the slots in free space and $Z_{0}$ is the free space impedance. The effect of the dielectric is negligible on the inductance $[16,22]$. The sub-dominant components of the circuit, namely $C_{\text {dipole }}$ and $L_{\text {slot }}$ can be estimated by adding the further Babinet's condition $\sqrt{C_{\text {dipole }} L_{\text {dipole }}}=1 / \omega_{0}$, where is the angular frequency at which a single layer of dipoles or slots resonates in free space, occurring when the length of the individual element is approximately half the wavelength. The value of $C_{1}$ plays a fundamental role in coupling and it is responsible for down-shifting the resonant frequency. Its value can be determined by numerical matching of the obtained final resonance. As a reference value, we may take the capacitance of a single dipole from (1); including the effect of the dielectric $C_{\text {ref }}=\left(\left(\varepsilon_{0}\left(\varepsilon_{\mathrm{r}}+1\right) p\right) / \pi\right) \ln ((1 / \sin (\pi p / 2 d)))$ where $\varepsilon_{\mathrm{r}}$ is the relative 


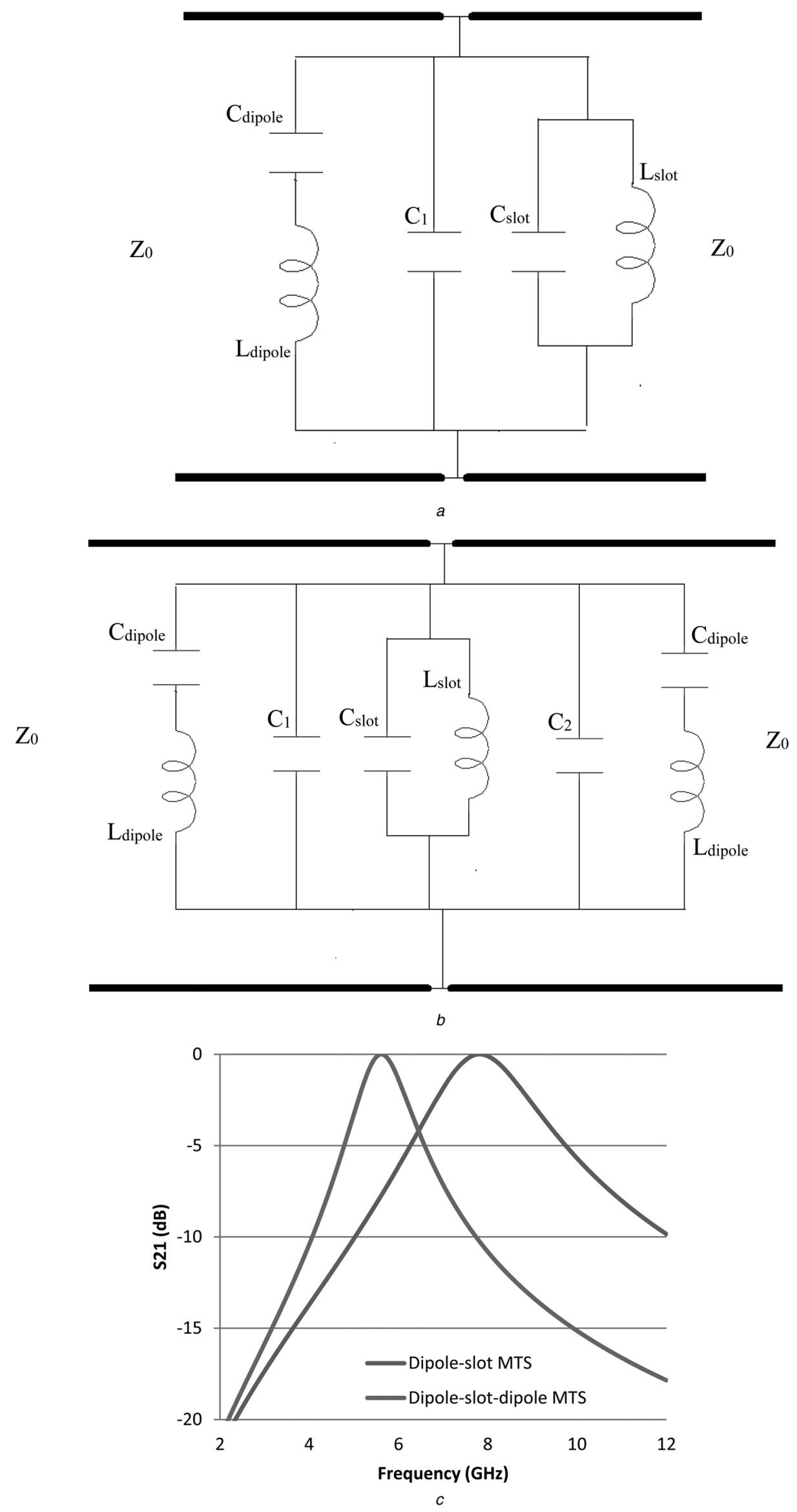

Fig. 4 Comparison between Equivalent Circuit Model of the dipole-slot MTS and the dipole-slot-dipole MTS

$a$ Equivalent circuit model for dipole-slot MTS

$b$ Equivalent circuit model for dipole-slot-dipole MTS

$c$ Equivalent circuit comparison (results) between the dipole-slot-dipole MTS and the dipole-slot MTS. The resonance of dipole-slot MTS was simulated to be $7.83 \mathrm{GHz}$, while dipoleslot-dipole MTS exhibited a resonance of $5.61 \mathrm{GHz}$ 


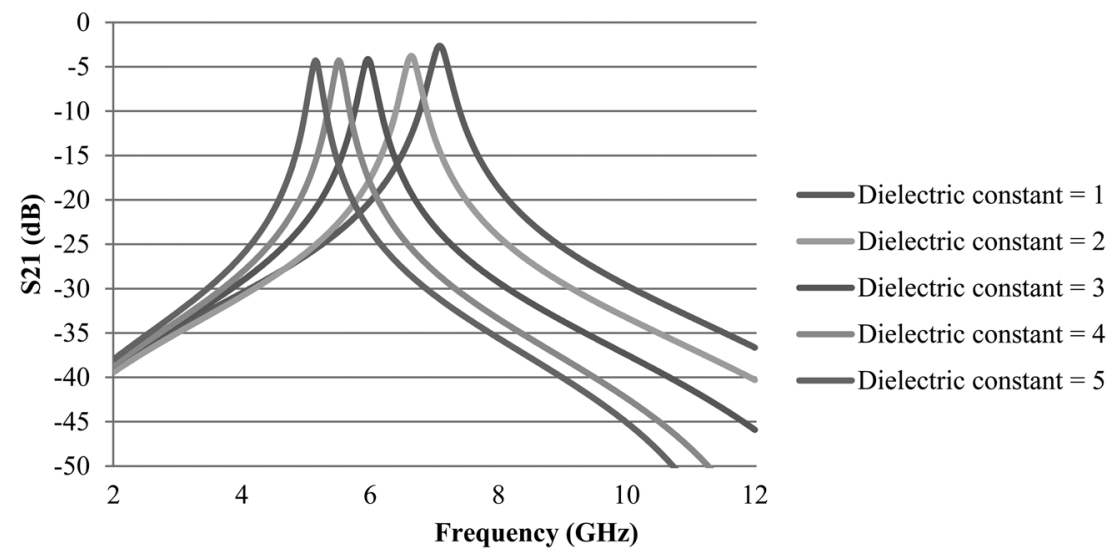

Fig. 5 Effect of changing the relative permittivity of the dielectric supporting the added dipole layer on the transmission response of dipole-slot-dipole MTS (simulated)

permittivity of the dielectric. We pose $C_{1}=n^{2} C_{\text {ref, }}$, where $n$ can be interpreted as a winding ratio of a transformer that reports capacitance at the slot level.

The equivalent circuit of the dipole-slot-dipole MTS is shown in Fig. $4 b$. It contains an extra coupling capacitance $C_{2}$ (which is equal to $C_{1}$ ), and another series $L C$ circuit representing the other dipole layer. The extra coupling capacitance is responsible is responsible for decreasing the resonant frequency with respect to the individual dipole-slot couple.

The results obtained with the previously described circuits are shown in Fig. 4c. The equivalent circuit response qualitatively predicts the one of the full-wave analysis with a value of $C_{1}=0.3$ $\mathrm{pF}$, which corresponds to $n \sim 2$; showing that the dipole capacitance has been quadrupled. This increase of capacitance is due to the strong concentration of electric fields around the zone of the slot-dipole crossing, which considerably increases the voltage across the slot (and then the winding ratio $n$ ) with respect to the voltage in isolation. The circuits have been simulated using AWR Microwave Office [23]. The values for the individual elements of the circuit are: $C_{\text {slot }}=0.0193 \mathrm{pF}, L_{\text {slot }}=1.17 \mathrm{nH}$, $C_{\text {dipole }}=0.033 \mathrm{pF}, L_{\text {dipole }}=0.688 \mathrm{nH}$. The equivalent circuit model assumes no losses in system.

Since the added capacitance, which arises due to the close proximity of the extra dipole layer, is the main reason for lowering the resonant frequency; the relative permittivity of the dielectric supporting the extra dipole layer will have a significant effect on the coupling. A higher value of the relative permittivity increases the added capacitance thus decreasing the resonant frequency. This is illustrated by varying the permittivity of the $44 \mu \mathrm{m}$ thick dielectric (supporting the third layer) in the simulations. The results for the simulations with different permittivities of the in-between dielectric are shown in Fig. 5 and summarised in Table 1.

The pass band resonance of the dipole-slot-dipole MTS decreases nearly linearly, from 7.09 to $5.15 \mathrm{GHz}$ as the relative permittivity of the dielectric sheet supporting the layer of dipoles is increased from 1 to 5 . It should be noted that even when the simulated material has a relative permittivity of 1 (equal to vacuum), the resonant frequency of dipole-slot-dipole MTS is still lower than the original resonant frequency of the dipole-slot MTS.

Table 1 Simulated change in the pass band resonance of dipole-slot-dipole MTS as the relative permittivity of the dielectric on the added dipole layer is varied
Relative permittivity of dielectric

supporting the dipole layer
Pass band resonant frequency, $\mathrm{GHz}$

\subsection{Effect of misalignment}

The electric fields across a slot vary as function of sine and are maximal at the centre of the slot; we expect a change of resonant frequency due to possible misalignment with respect to the optimal centred configuration. In order to investigate the effect of this misalignment, the added dipole array was analysed for the gradual shifting away from the centre of the slot. It was observed that as the dipole array (third layer) was moved away from the centre, the resonant frequency of dipole-slot-dipole MTS increased. These results are shown in Fig. 6.

When the centre of the dipole array was aligned with the centre of the slot array, the resonant frequency of the slot-dipole-slot MTS was $5.96 \mathrm{GHz}$, but as it was moved $0.5 \mathrm{~mm}$ away, the resonant frequency increased to $6.33 \mathrm{GHz}$. The resonant frequency was 6.73 and 7.18 $\mathrm{GHz}$ when the distance between the centre of the dipole and the centre of the slot was 1 and $1.5 \mathrm{~mm}$, respectively. This shows that the maximum coupling takes place when the centre of the dipoles and the slots are aligned.

For practical purposes, this highlights the fact that the alignment of the both layers is very important and must be given careful consideration, as a misalignment of only $0.5 \mathrm{~mm}$ of the dielectric backed dipole layer away from the centre can cause an upward shift of the pass band resonance.

\subsection{Effects of variation of length of dipoles}

The dipole-slot-dipole MTS is a $\lambda / 10$ periodic structure at resonance, which makes it extremely compact as compared to a traditional frequency selective surface. This makes the stand-alone dipole

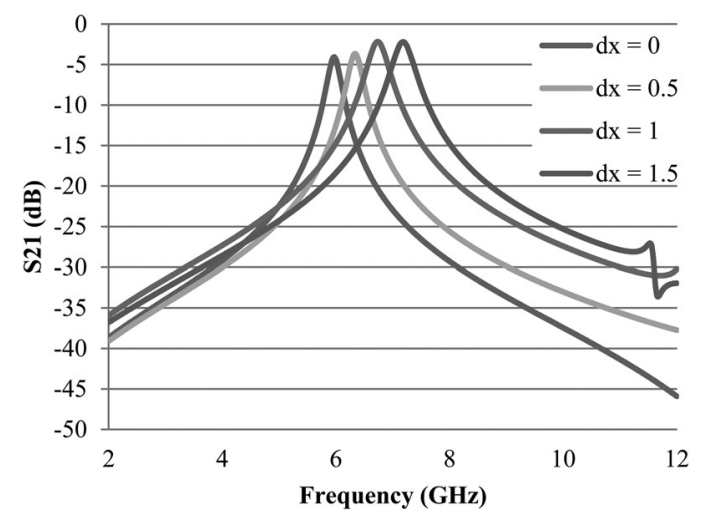

Fig. 6 Simulated pass band resonance of the dipole-slot-dipole MTS as the function of the relative position $(\mathrm{dx}(\mathrm{mm}))$ of the added dipole as compared with the slot 


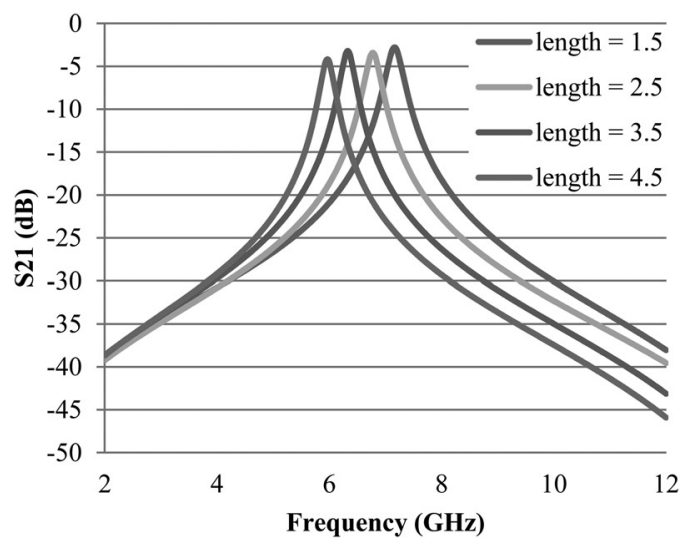

Fig. 7 Simulated effect of changing the length of the dipole ( $\mathrm{mm}$ ) on the transmission characteristics of dipole-slot-dipole MTS

layer transparent at the resonant frequency when a stand-alone layer of slots behaves like a perfect reflector. This also shows that the response of the dipole-slot-dipole MTS is mainly governed by the gap-coupling among the elements present on different layers; therefore the extra capacitance added to the original dipole-slot MTS leads to a significant decrease in its resonant frequency. The response of the dipole-slot-dipole MTS to changing the length of the dipole placed on the added dielectric backed layer (third layer) has also been investigated. The thickness of the whole structure is very small as compared with the wavelength, while the coupling among the adjacent layers is very strong. This makes the dipole-slot-dipole MTS behave like a single pass band structure even after breaking the geometrical symmetry. The length of one dipole layer was varied from to 4.5 to $1 \mathrm{~mm}$ showing a decreased in the resonant frequency of the structure. This is attributed to the fact that as the size of the dipole is decreased, the added capacitance also decreases, which reduces the coupling between the added dipole layer and the dipole-slot MTS. The results are shown in Fig. 7.

As the length increases from 1.5 to $4.5 \mathrm{~mm}$ with an increment of 1 $\mathrm{mm}$, the frequency increases from $5.96 \mathrm{GHz}$ to $6.33,6.77$ and 7.16 $\mathrm{GHz}$, respectively.

\section{Experimental verification}

The dipole-slot MTS samples were fabricated by etching the designs onto a thin GTS laminate with metal cladding on both sides. The thickness of the dielectric was $44 \mu \mathrm{m}$ and the relative permittivity of GTS is equal to 3 [24]. The sample size was designed to be

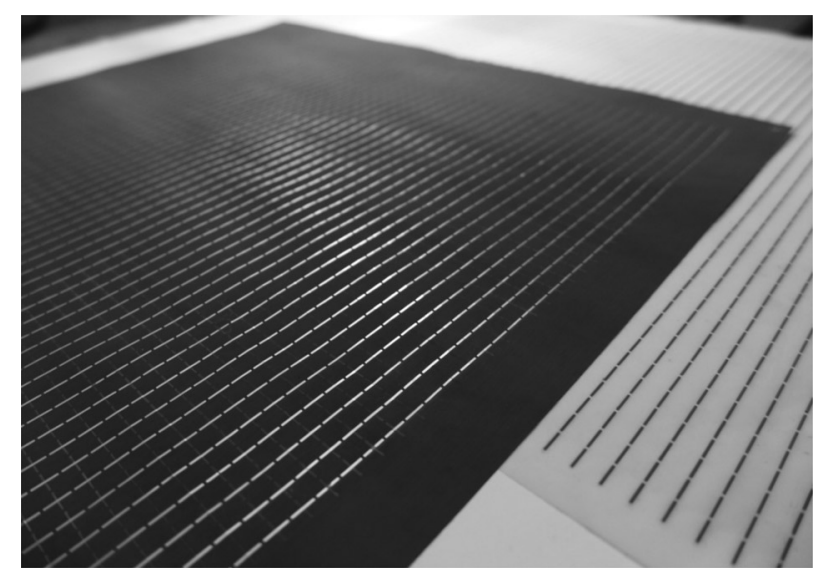

Fig. 8 Fabricated samples showing a dipole-slot MTS and an additional layer of dipoles (third layer). The dipoles and slots are $4.5 \mathrm{~mm}$ long and $0.5 \mathrm{~mm}$ wide with a periodicity of $5 \mathrm{~mm}$

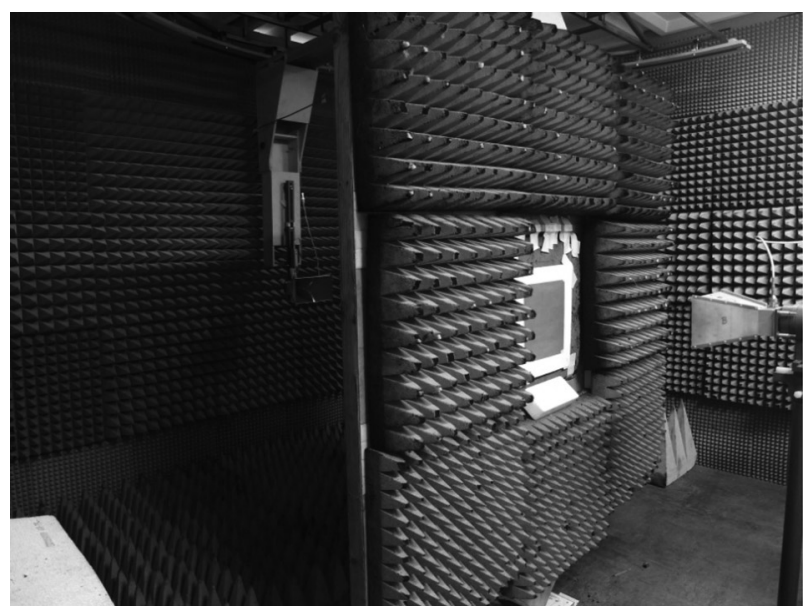

Fig. 9 Measurement setup of MTSs between two horn antennas

$260 \times 260 \mathrm{~mm}^{2}$. This size allowed an array of $52 \times 52$ elements. In order to construct a dipole-slot-dipole MTS, another layer of dipoles, backed by the same $44 \mu \mathrm{m}$ thick GTS was also fabricated. The dipoles were etched on its one side, while the metal was completely removed from the other. Fig. 8 shows the fabricated samples.

The pass band and the stop band were measured in a semi-anechoic chamber. Two horn antennas operating from 2 to $12 \mathrm{GHz}$, which were placed $3.5 \mathrm{~m}$ apart. The incident $E$-field was vertical across the slot. The surfaces were placed in a $250 \times 250$ $\mathrm{mm}^{2}$ square aperture in the RAM wall. The measurement setup is shown in Fig. 9.

The dipole-slot-dipole MTS was constructed by placing the GTS-backed dipole layer on the top of the dipole-slot MTS. As shown in Section 2.3, the dipole-slot-dipole MTS is sensitive to the alignment of the dipoles so careful consideration was given in aligning the two layers. Both layers were sandwiched between two sheets of Rohacell and were tightly pressed against each other in order to avoid any air gap as that can lead to the reduction in the added capacitance, which manifests itself in increasing the resonant frequency of the dipole-slot-dipole MTS. The samples were positioned in the far field of the horn antennas. The results shown are normalised compared with the received power without the samples. Note, the samples are polarisation sensitive and the dipoles were orientated vertically so as to be parallel with the polarisation of the horn antennas. The comparisons between the measured and simulated results for both MTSs are shown in Figs. 10 and 11. A very good agreement between the simulated and the measured results was observed.

The comparisons between the measured transmission response of the dipole-slot MTS and the dipole-slot-dipole MTS is shown in

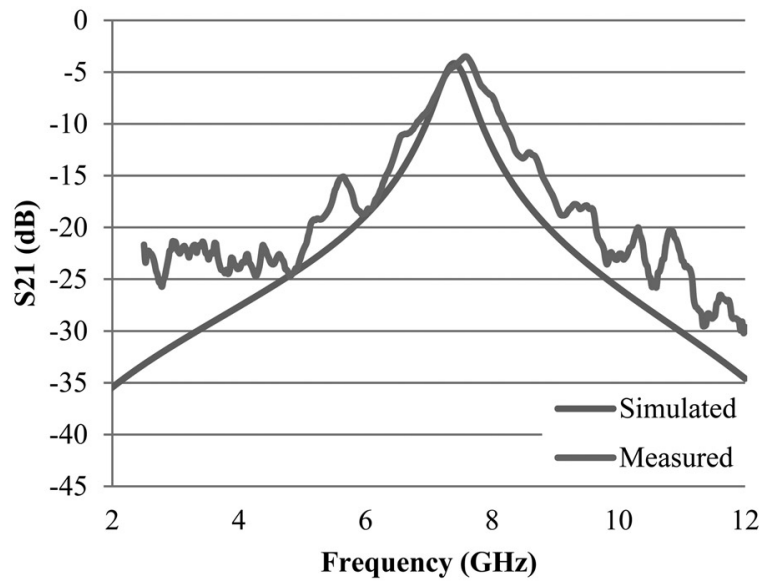

Fig. 10 Measured and simulated response of dipole-slot MTS 


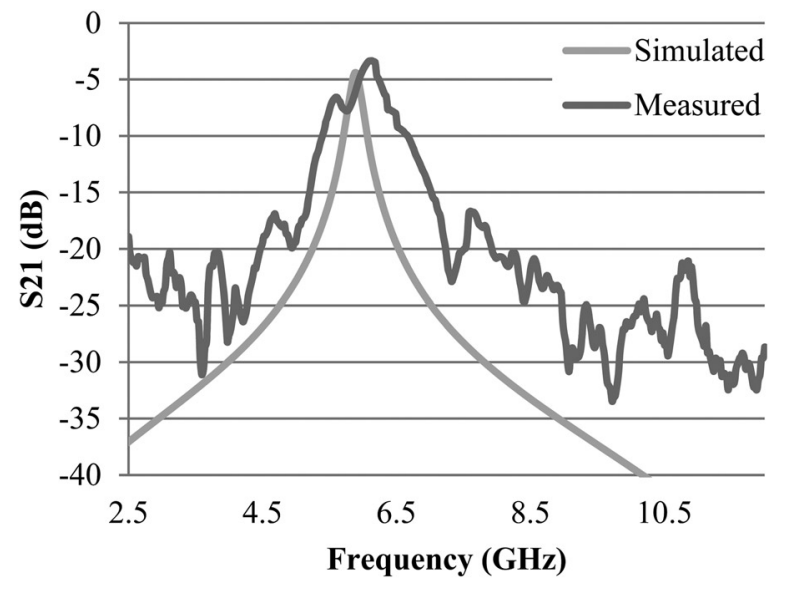

Fig. 11 Measured and simulated response of dipole-slot-dipole MTS

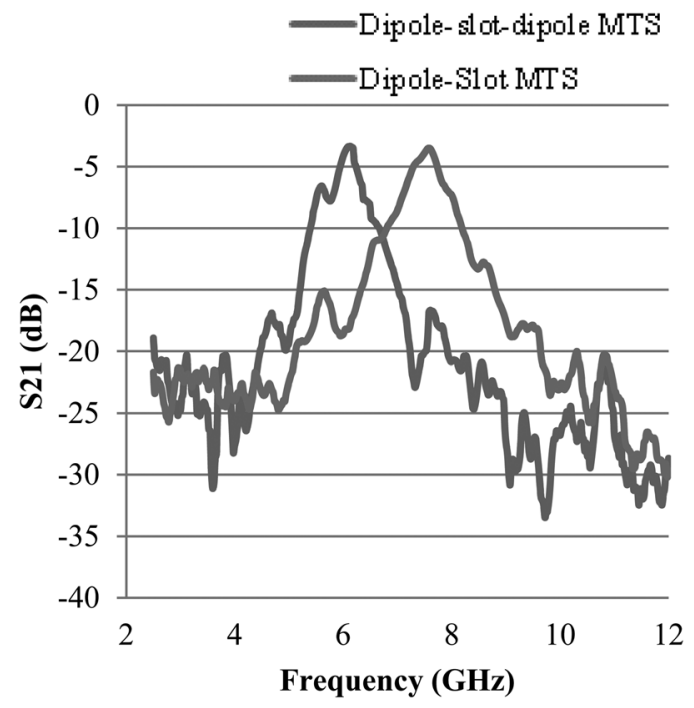

Fig. 12 Comparison of measured S21 of dipole-slot-dipole MTS and dipole-slot MTS

Fig. 12. This comparison shows that by adding a layer of dipoles to the dipole-slot MTS, the resonant frequency of the structure has been decreased. Slight differences between measured and simulated values for the dipole-slot-dipole MTS can be attributed to mis-alignment.

\section{Conclusions}

The behaviour of the dipole-slot MTS is controlled by the strong coupling among its layers. This property allows us to miniaturise this structure by adding extra capacitance. This extra capacitance was added by adjacently placing a layer of dipoles and resulted in decreasing the pass band resonant frequency of the structure form 7.5 to $6.1 \mathrm{GHz}$. An equivalent circuit model has been presented. This model qualitatively explains the behaviour of dipole-slot-dipole MTS and also illustrates its comparison with dipole-slot MTS. It should be noted that due to very strong coupling between the layers, the loss in both structures is closely related to the properties of the dielectric material. The new structure has a period of one-tenth of the free-space wavelength at the resonant frequency. It is called a dipole-slot-dipole MTS. Since the miniaturisation is the result of adding extra capacitance, it was shown that increasing the relative permittivity of the material supporting the dipoles array led to a decrease in the resonant frequency. Decreasing the length of the added dipoles causes a decrease in this capacitance, therefore increasing the resonant frequency of the dipole-slot-dipole MTS. The electric fields of the slot are maximal at its centre and it was demonstrated that the maximum coupling between the dipole-slot MTS and the dielectric backed dipole layer occurs when the centre of the dipoles overlap the centre of the slots. A lack of alignments decreases the coupling and causes an increase in the pass band resonance. The experimental validation was carried out by fabricating both, the dipole-slot MTS and dipole-slot-dipole MTS, and their response to an incoming plane wave was measured in a semi-anechoic chamber. A very good agreement is found between the simulations and the measured results.

\section{References}

1 Holloway, C.L., Kuester, E.F., Gordon, J.a., et al.: 'An overview of the theory and applications of metasurfaces: the two-dimensional equivalents of metamaterials', IEEE Antennas Propag. Mag., 2012, 54, (2), pp. 10-35

2 Holloway, C.L., Kuester, E.F., Dienstfrey, A.: 'Characterizing metasurfaces/ metafilms: the connection between surface susceptibilities and effective material properties', IEEE Antennas Wirel. Propag. Lett., 2011, 10, pp. 1507-1511

3 Holloway, C.L., Kuester, E.F., Novotny, D.: 'Waveguides composed of metafilms/ metasurfaces: the two-dimensional equivalent of metamaterials', IEEE Antennas Wirel. Propag. Lett., 2009, 8, pp. 525-529

4 Jiang, Z.H., Brocker, D.E., Member, S., et al.: 'A compact low-profile metasurface-enabled network devices', IEEE Trans. Antennas Propag., 2014, 62, (8), pp. 4021-4030

5 Huang, C., Pan, W., Ma, X., et al.: 'Wideband radar cross-section reduction of a stacked patch array antenna using metasurface', IEEE Antennas Wirel. Propag. Lett., 2015, 14, (c), pp. 1369-1372

6 Minatti, G., Caminita, F., Casaletti, M., et al.: 'Spiral leaky-wave antennas based on modulated surface impedance', IEEE Trans. Antennas Propag., 2011, 59, (12), pp. 4436-4444

7 Gregoire, D.J.: '3-D conformal metasurfaces', 2013, 12, pp. 233-236

8 Konstantinidis, K., Feresidis, A.P., Hall, P.S.: 'Broadband sub-wavelength profile high-gain antennas based on multi-layer metasurfaces', IEEE Trans. Antennas Propag., 2015, 63, (1), pp. 423-427

9 Sievenpiper, D.F.: 'Nonlinear grounded metasurfaces for suppression of high-power pulsed RF currents', IEEE Antennas Wirel. Propag. Lett., 2011, 10, pp. 1516-1519

10 Yu, N., Capasso, F.: 'Optical metasurfaces and prospect of their applications including fiber optics', J. Lightwave Technol., 2015, 33, (12), pp. 2344-2358

11 Ra, Y., Tretyakov, S.A.: 'Angularly-independent Huygens metasurfaces'. 2015 IEEE Int. Symp. Antennas and Propagation \& USNC/URSI National Radio Science Meeting, 2015, pp. 874-875

12 Selvanayagam, M., Member, S., Eleftheriades, G.V.: 'Circuit modeling of Huygens surfaces', 2014, 12, (2), p. 1642-1645

13 Wu, Q., Scarborough, C.P., Werner, D.H., et al.: 'Design synthesis of metasurfaces for broadband hybrid-mode horn antennas with enhanced radiation pattern and polarization characteristics', IEEE Trans. Antennas Propag., 2012, 60, (8), pp. 3594-3604

14 Wu, Q., Scarborough, C.P., Martin, B.G., et al.: 'A Ku-band dual polarization hybrid-mode horn antenna enabled by printed-circuit-board metasurfaces', IEEE Trans. Antennas Propag., 2013, 61, (3), pp. 1089-1098

15 Lockyer, D.S., Vardaxoglou, J.C., Simpkin, R.A.: 'Complementary frequency selective surfaces', IEE Proc Microw. Antennas Propag., 2000, 147, (6), pp. $501-5-7$

16 González-Ovejero, D., Martini, E., Maci, S.: 'Surface waves supported by metasurfaces with self-complementary geometries', IEEE Trans. Antennas Propag., 2015, 63, (1), pp. 250-260

17 Ortiz, J.D., Baena, J.D., Losada, V., et al.: 'Self-complementary metasurface for designing narrow band pass/stop filters', IEEE Microw. Wirel. Compon. Lett., 2013, 23, (6), pp. 291-293

18 Gonzalez-Ovejero, D., Martini, E., Loiseaux, B. et al. 'Basic properties of checkerboard metasurfaces', IEEE Antennas Wirel. Propag. Lett., 2015, 14 pp. 406-409

19 Bukhari, S.S., Whittow, W.G., Vardaxoglou, J.Y.C., et al.: 'Dipole and slot loaded closely coupled complementary metasurfaces'. Loughborough Antennas and Propagation Conf., 2015, pp. 7-9

20 Ramaccia, D., Toscano, A., Bilotti, F.: 'A new accurate model of high-impedance surfaces consisting of circular patches', Prog. Electromagn. Res. M, 2011, 21, (May), pp. 1-17

21 Luukkonen, O., Simovski, C., Granet, G., et al.: 'Simple and accurate analytical model of planar grids and high-impedance surfaces comprising metal strips or patches', IEEE Trans. Antennas Propag., 2008, 56, (6), pp. 1624-1632

22 Sarabandi, K., Behdad, N.: 'A frequency selective surface with miniaturized elements', IEEE Trans. Antennas Propag., 2007, 55, (5), pp. 1239-1245

23 'Microwave Office: 'http://www.awrcorp.com/products/microwave-office'

24 Njoku, C.C., Whittow, W.G., Vardaxoglou, J.C.: 'Effective permittivity of heterogeneous substrates with cubes in a 3-D Lattice', IEEE Antennas Wirel. Propag. Lett. (Spec. Issue Metamater.), 2011, 10, pp. 1480-1483 